\title{
La relación entre la patente y el desarrollo: Una mirada desde la $\mathrm{I}+\mathrm{D}$ universitaria chilena
}

\author{
The relationship between the patent and development: \\ $A$ view from chilean academic R\&D
}

\author{
Sulan Wong \\ Universidad de La Frontera, Chile \\ Julio Rojas-Mora \\ Universidad Católica de Temuco, Chile
}

\begin{abstract}
RESUMEN Al declarar que el Objetivo de Desarrollo Sostenible 9 activa otros de los diecisiete objetivos propuestos en 2015 por Naciones Unidas en esta materia, la Organización Mundial de la Propiedad Intelectual induce a que los gobiernos incentiven la relación entre la universidad y la industria, como principales productoras de resultados de investigación patentables. A partir de datos recogidos entre 2017 y 2018 en las siete universidades que más patentan en Chile, encontramos que, primero, existen relaciones entre los entrevistados y la industria; segundo, la mayoría de los entrevistados ha patentado; tercero, estas patentes no surgen de proyectos industria-universidad; y cuarto, la patente no se percibe como un incentivo fundamental para el desarrollo. Por tanto, el modelo chileno de producción de investigación y desarrollo (I+D) parece más lineal que de triple hélice.
\end{abstract}

PALABRAS CLAVE Patente, I+D, desarrollo, modelo lineal de innovación, modelo de innovación de triple hélice.

ABSTRACT By declaring that Sustainable Development Goal 9 activates others of the seventeen goals adopted by the United Nations General Assembly in 2015 on this regard, the World Intellectual Property Organization encourages governments to promote the relationship between the university and industry as the primary producers of patentable research results. Based on data collected between 2017-2018 in the seven universities that patent the most in Chile, we find that, first, there are relationships between the interviewees and the industry; second, most of the interviewees have patented; third, these patents do not arise from industry-university projects; and fourth, patenting is 
not perceived as a fundamental activity for development. Therefore, the Chilean R\&D production model appears linear rather than triple helix.

KEYWORDS Patent, R\&D, development, linear innovation model, triple helix innovation model.

\section{Introducción}

La Agenda 2030 sobre el Desarrollo Sostenible enumera 17 objetivos de desarrollo sostenible (ODS) (Organización de las Naciones Unidas, 2015) que nos conminan a abandonar el estado de indiferencia en el que nos encontramos frente a problemas globales como el hambre y la pobreza, la contaminación ambiental o la desigualdad en el acceso a los servicios sanitarios y educativos. En este sentido, la Organización Mundial de la Propiedad Intelectual (OMPI, 2018) sostiene que el ODS 9 - asociado a la industria, innovación e infraestructura - «es la base misma de nuestra misión para liderar el desarrollo del sistema internacional de propiedad intelectual en beneficio de todos»; lograr este objetivo es esencial para alcanzar los ODS 2 (Hambre Cero), 3 (Salud y Bienestar), 6 (Agua Limpia y Saneamiento), 7 (Energía Asequible y No Contaminante), 8 (Trabajo Decente y Crecimiento Económico), 11 (Ciudades y Comunidades Sostenibles) y 13 (Acción por el Clima) (OMPI, 2018). Se puede entender de este mensaje el discurso de la relación causal entre la propiedad intelectual y el desarrollo, mediado por el desarrollo industrial. Este discurso es presentado en un interesante artículo publicado recientemente por el profesor Peter Yu (2016), quien señala que, en las últimas cinco décadas, la OMPI ha desplegado un amplio abanico de iniciativas pro desarrollo vinculadas a las normas y políticas de propiedad intelectual. No obstante, la entrada en vigencia de los ODS invita a la reflexión sobre dicho discurso; los países en desarrollo presionan por una interpretación menos restrictiva de la relación entre los ODS y la propiedad intelectual y los países desarrollados no comparten este enfoque.

El argumento presentado por los países en desarrollo puede verse, por ejemplo, en el ODS 3 b que expresamente señala la relación entre el derecho a la salud y el acceso a medicamentos, apoyando «el derecho de los países en desarrollo a utilizar al máximo las disposiciones del Acuerdo sobre los Aspectos de los Derechos de Propiedad Intelectual Relacionados con el Comercio en lo relativo a la flexibilidad para proteger la salud pública y, en particular, proporcionar acceso a los medicamentos para todos» (Organización de las Naciones Unidas, 2015). Por otra parte, la estrategia de inversión en los países en desarrollo considerada en los ODS estaría basada en la inversión en ciencia interdisciplinaria de desarrollo sostenible, la que fomentará la reducción de la pobreza y la contaminación ambiental (Sachs y Reid, 2006). 
La investigación y desarrollo (I+D) está comprendida entre las actividades científicas y tecnológicas (ACT) definidas por la Unesco (1978). La OCDE la diferencia de otras actividades científicas, tecnológicas e industriales por «la existencia en el seno de la I+D de un elemento apreciable de novedad y resolución de incertidumbre científica y/o tecnológica» (OCDE, 2002: \$84). Esta diferenciación, apoyada por criterios complementarios, ${ }^{1}$ permite la identificación de la investigación cuyos resultados pueden ser objeto de solicitud de patente. Como el financiamiento de cualquier tipo - público, privado o mixto- de la investigación científica suele etiquetarse como «I+D», parece claro que la $\mathrm{I}+\mathrm{D}$ es el elemento retórico que justifica la apropiación y mercantilización de la ciencia a través de los derechos de propiedad intelectual, que los agentes privados defienden con el acuerdo estatal y académico.

El lucro es la función objetivo más importante y apropiada para la industria, mientras que las universidades son las organizaciones sociales de facto que fueron originalmente creadas para el fomento del bien público (Hayter, 2016). No obstante, Van Overwalle (2006) expone acerca de la creciente presión sobre las universidades para sacar provecho del potencial comercial creado por su investigación. Tanto las universidades como los individuos académicos son cada vez más propensos a considerar sus conocimientos como objetivos de oportunidades para generar ingresos; a la universidad se le ha asignado una nueva misión, la transferencia del conocimiento.

El discurso de la transferencia de conocimiento científico alcanzado por las universidades y centros de investigación mediante la patente, es decir, privatizándolo para comercializarlo, es - por qué no dejarlo claro- irónico, dado el carácter sin fines de lucro que las instituciones de educación superior suelen tener en el mundo, ${ }^{2}$ lo que las hace acreedoras de exenciones fiscales sobre los beneficios económicos de

\footnotetext{
1. Véase Cuadro 2.1 en OCDE (2002).

2. Esto no las blinda en todos los casos. Por ejemplo, la Corte del Circuito Federal del Tercer Distrito de los Estados Unidos rechazó el fallo de la Corte de Distrito del Distrito Medio de Carolina del Norte en el famoso caso Madey con Duke, reconociendo que el carácter sin fin de lucro de la Universidad de Duke no la acreditaba a utilizar libre y gratuitamente, para fines de investigación, las invenciones patentadas por el Dr. John Madey; por el contrario, dado que con los resultados de esas investigaciones la Universidad de Duke incrementaría su prestigio, eso le permitiría conseguir mejores académicos, mejores financiamientos y mejores estudiantes, lo que en suma implicaría obtener una utilidad de su legítimo negocio, independientemente de que esta utilidad fuese pecuniaria o no. Véase Madey con Duke University, 307 F.3d 1351 (Fed. Cir., 2002). Esta decisión es, cuando menos, peligrosa para las universidades que realizan investigación en países donde la excepción de investigación no ha sido integrada en su marco jurídico. Éste era el caso en Chile, aunque la excepción de investigación se ha incluido en la Ley 19.039 de Propiedad Industrial a través de dos literales del último inciso del artículo 49: «El derecho conferido por la patente no se extenderá: a) A los actos realizados privadamente y sin motivos comerciales, b) A los actos realizados por motivos exclusivamente experimentales relativos al objeto de la invención patentada». Habrá que ver como interpretarán los tribunales del país ambas formas de excepción.
} 
sus actividades directas ${ }^{3} \mathrm{y}$ de fondos para el financiamiento de la investigación científica y tecnológica.

El objetivo de este trabajo es, en primer término, presentar un panorama de la relación industria-universidad en la $\mathrm{I}+\mathrm{D}$ realizada en el contexto de la academia chilena, mediada por la patente como instrumento de transferencia tecnológica y su capacidad de fomentar el desarrollo. Para esto, presentamos un marco teórico en el que estudiamos los dos principales modelos de innovación ${ }^{4}$ cubiertos por la literatura especializada y que dan cuenta de los actores de interés para este estudio: el modelo lineal y el modelo de triple hélice. A continuación, presentaremos la metodología utilizada para la recolección y análisis de datos. Continuaremos con los resultados alcanzados, para cerrar con un apartado de discusión y conclusiones.

\section{Marco teórico}

\section{El modelo lineal de innovación}

La OCDE define la I+D como «el trabajo creativo llevado a cabo de forma sistemática para incrementar el volumen de conocimientos, incluido el conocimiento del hombre, la cultura y la sociedad, y el uso de esos conocimientos para crear nuevas aplicaciones». ${ }^{5}$ La sigla I+D, que comenzó a usarse a partir de los años cuarenta del siglo pasado, engloba tres actividades específicas de investigación: investigación básica, investigación aplicada y desarrollo. Se encuentra en esta definición el modelo lineal de innovación que hace énfasis en el flujo unidireccional de la ciencia hacia la ingeniería y así sucesivamente hacia la producción y el mercado. El modelo lineal

3. En Chile, el Servicio de Impuestos Internos reconoce que «los ingresos percibidos por las universidades estatales o por aquellas reconocidas por el Estado, proveniente únicamente de las actividades de educación superior que imparten tales entidades, se encuentran exentos del Impuesto de Primera Categoría, conforme a lo dispuesto en el artículo único de la Ley 13.713, de 1959, en concordancia con lo establecido por el artículo 14 del DL 1.604, de 1976». Al respecto, véase lo dispuesto por el SII en el siguiente enlace: https://bit.ly/3F9ETDs. No obstante, considera que «de acuerdo a la letra h), del artículo 8, de la Ley sobre Impuesto a las Ventas y Servicios, que el arrendamiento, subarrendamiento, usufructo o cualquiera otra forma de cesión del uso o goce temporal de patentes de invención, entre otros, se grava con el impuesto al valor agregado, salvo que las rentas respectivas se afecten con el impuesto adicional establecido en el artículo 59 de la Ley sobre Impuesto a la Renta, caso en el cual se eximirían de dicho impuesto indirecto, de conformidad a lo previsto en el número 7, de la letra e), del artículo 12 de la Ley sobre Impuesto a las Ventas y Servicios». Véase Servicio de Impuestos Internos (2016).

4. Para indagar sobre otros modelos de innovación, como el de cuádruple hélice y quíntuple hélice, recomendamos ver el trabajo de Carayannis y otros (2017).

5. Véase OCDE (2002: 30). Esta definición de I+D coincide con la elaborada por la Unesco: investigación científica y desarrollo experimental es «cualquier trabajo sistemático y creador realizado con el fin de aumentar el caudal de conocimientos, inclusive el conocimiento del hombre, la cultura y la sociedad, y de utilizar esos conocimientos para descubrir nuevas aplicaciones». Véase Unesco (1978: § 2.1 a). 
sugiere que la innovación comienza con la investigación básica, a la que le sigue la investigación aplicada y el desarrollo, culminando con la producción y difusión. Se abandona con este modelo la vieja concepción dicotómica de presentar a la investigación básica frente a la investigación aplicada, mostrándolas más bien como actividades secuenciales.

Tal y como Leyden y Menter (2018) apuntan, la investigación básica se centraría en el descubrimiento de nuevos conocimientos fundamentales y maximizaría su utilidad al no estar restringida por controles externos ni depender de los recursos de quienes tienen intereses más inmediatos y prácticos. El nuevo conocimiento fundamental que se crea a través de la investigación básica proporcionaría la base para la investigación aplicada, la que, mediante sus resultados de investigación de utilidad práctica, sirve como base para proyectos de desarrollo y, en última instancia, para la generación de nuevos productos y el empleo de nuevos métodos de producción. Bajo este modelo los indicadores de la ciencia son constructos útiles para establecer políticas de inversión de acuerdo a la motivación subyacente de la investigación (Murray y Stern, 2007: 63).

El diagrama del modelo tradicional de innovación (Siegel y otros, 2004, modificado por Bradley, Hayter y Link, 2013) da cuenta del modelo lineal, aunque asume, de la misma forma en que lo hace la Ley Bayh-Dole de los Estados Unidos (Leyden y Link, 2015: 80), que la investigación básica ha sido ya realizada y sirve de base a su punto de inicio: el descubrimiento por un investigador universitario de un resultado potencialmente patentable (figura 1). En este modelo es este investigador quien activa el proceso, al revelar a la Oficina de Transferencia Tecnológica (OTT) de su universidad dicho resultado. Esta, luego de evaluar la factibilidad de obtener una patente sobre el mismo y de introducirla al mercado exitosamente, decide si procede a redactar y elevar una solicitud de patente o si devuelve el resultado al investigador para que este haga con él lo que considere adecuado (publicarlo, habitualmente, aunque podría decidir arriesgarse y buscar un socio comercial para solicitar una patente). Una vez que la universidad solicita y obtiene una patente sobre el resultado de investigación, procede a colocar en el mercado la invención, buscando empresas que pudieran estar interesadas en licenciarla para fabricarla y comercializarla. Cuando se llega a un acuerdo con una empresa sobre los términos de la licencia, el paso final es decidir si la empresa será la encargada de la fabricación y comercialización de la invención o si, por el contrario, será una spin-off (una escisión de la empresa licenciataria o de la universidad, especializada en el producto patentado) o una start-up (una nueva empresa con participación mixta entre la universidad, la empresa licenciataria e inversionistas de riesgo) la que lo hará.

Ziman (2002: 15) observa que este modelo es reduccionista, pero que es subyacente a la forma de entender la «ciencia» que tienen los políticos, los empresarios, los funcionarios públicos y hasta la prensa. Para Godin (2006), esta construcción teoré- 


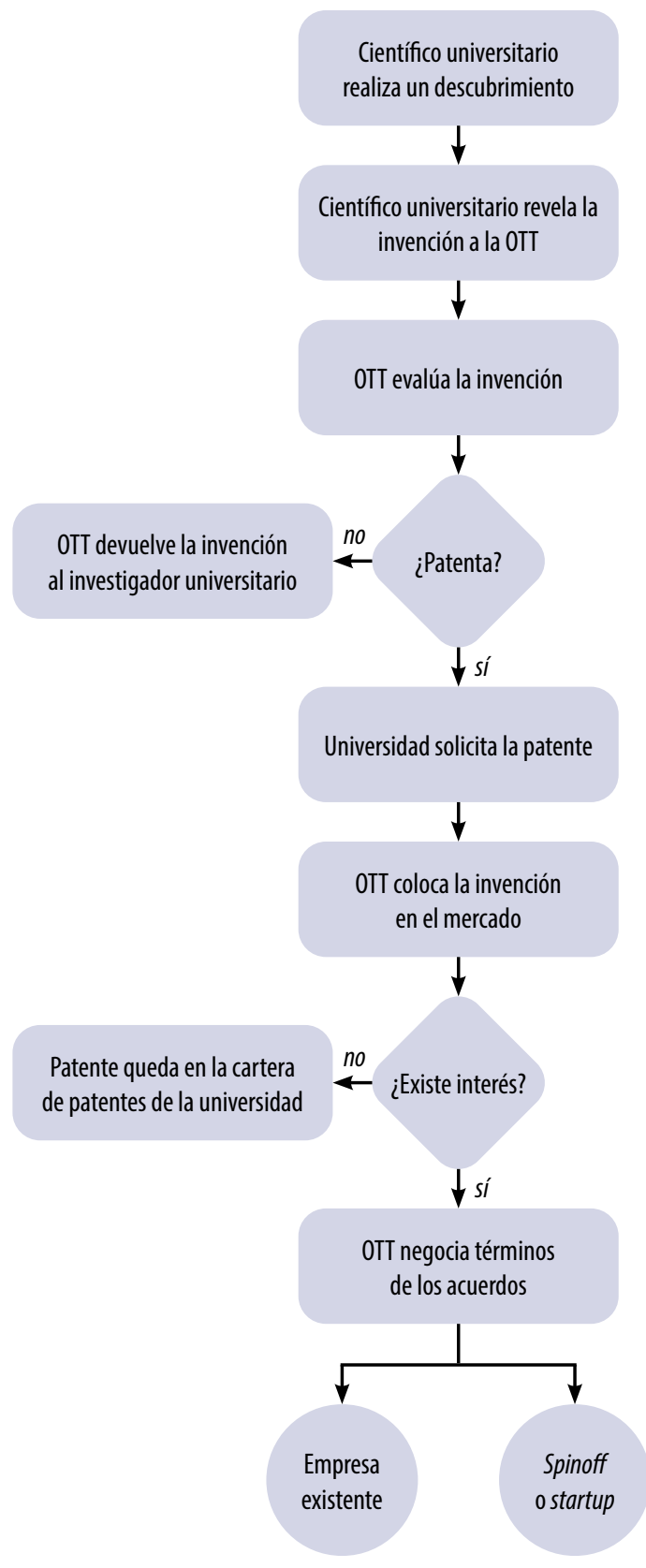

Figura 1. Modelo lineal de innovación de Siegel y otros (2004), modificado por Bradley, Hayter y Link (2013). 
tica, localizada en la retórica política, puede atribuirse a industriales, consultores y escuelas de negocios, secundados por economistas. La OCDE, por su parte, advierte que problemas prácticos y teóricos asociados a las tres actividades del modelo lineal de $\mathrm{I}+\mathrm{D}$, impiden en la realidad establecer una separación entre las mismas: la $\mathrm{I}+\mathrm{D}$ puede darse en un mismo centro y ser prácticamente realizada por el mismo personal; incluso el progreso puede ser bidireccional, y algunos proyectos de investigación pueden estar a la vez en fase de investigación básica y aplicada. ${ }^{6}$ Además, durante la fase de comercialización de la invención, es común que no solo la universidad como socia participe, sino que el investigador continúe relacionado para ayudar a desarrollar el producto final (Bradley, Hayter y Link, 2013; Thursby, Jensen y Thursby, 2001).

\section{El modelo de innovación de «triple hélice»}

El modelo alternativo al de innovación lineal es el llamado «modelo de triple hélice», en el que convergen la universidad, la industria y el Gobierno para implementar proyectos de I+D (Etzkowitz y Leydesdorff, 200o). El Gobierno cofinancia, junto a la industria, estos proyectos, definiendo grosso modo las líneas de investigación prioritarias para el desarrollo del país, pero dejando a la industria la decisión final sobre los proyectos que apoyará. Este modelo ha evolucionado a lo largo de tres fases (figura 2). La triple hélice I es de tipo estatista, ya que el Gobierno controla las relaciones entre la industria y la universidad, forzando su colaboración. Es un modelo considerado como fallido, pues obstaculiza las iniciativas ascendentes y la innovación es desalentada. La triple hélice II es un modelo de tipo laissez faire, en el que cada actor tiene una esfera separada de acción, estableciendo relaciones según sea conveniente, siendo antitético al primero, pues es planteado para reducir la intervención del Gobierno. El modelo de triple hélice III es un modelo sintético de los otros dos, pues busca puntos de encuentro entre los tres actores, aunque también deja espacio para relaciones entre dos de ellos y para la acción individual. Este modelo, de acuerdo a Etzkowitz y Leydesdorff (2000), es el que persiguen la mayoría de los países, pues sus gobiernos alentarían la relación industria-universidad, pero no necesariamente

6. Reproducimos aquí uno de los ejemplos con que el Manual de Frascati intenta ilustrar las diferencias generales entre las actividades $\mathrm{I}+\mathrm{D}$ de las ciencias exactas, naturales e ingeniería: la determinación de la secuencia de aminoácidos de una molécula anticuerpo es investigación básica. La misma investigación, emprendida en un esfuerzo por distinguir entre anticuerpos de diversas enfermedades, es investigación aplicada. El desarrollo experimental sería, por ejemplo, la búsqueda de un método para sintetizar el anticuerpo de una enfermedad determinada basándose en el conocimiento de su estructura, así como el conjunto de ensayos clínicos correspondientes para determinar la efectividad del anticuerpo sintetizado en pacientes que hayan aceptado someterse, a título experimental, a ese tratamiento de vanguardia (OCDE, 2002: §253). 

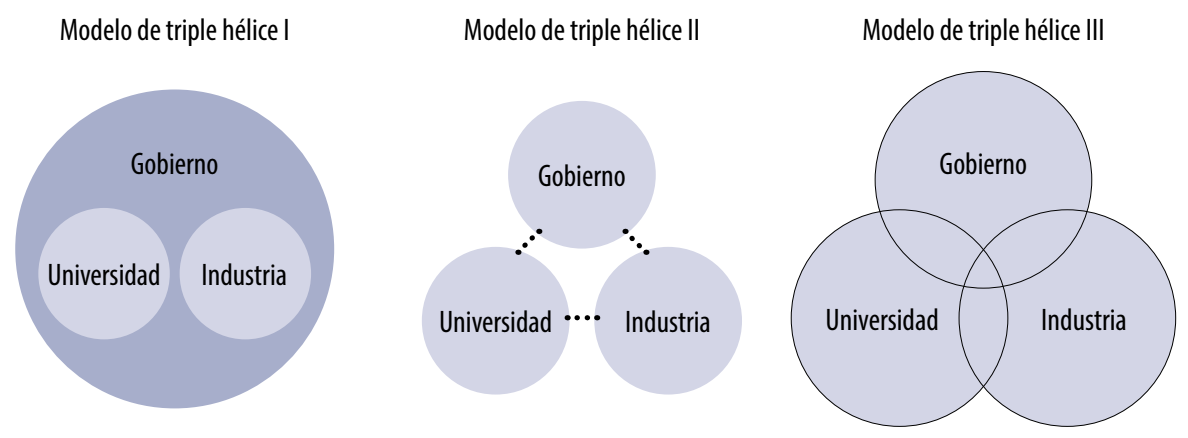

Figura 2. Modelo de triple hélice de Etzkowitz y Leydesdorff (2000).

la controlarían.7 El ejemplo paradigmático de este tipo de relación es la establecida en Estados Unidos mediante la Ley Bayh-Dole, con la que se alentaba a la industria y a la universidad a compartir los beneficios económicos de los resultados de investigación de proyectos financiados con fondos públicos mediante la privatización conjunta de los mismos.

Chile, con la promulgación de la Ley 21.105, que crea el Ministerio de Ciencia, Tecnología, Conocimiento e Innovación, se introduce en esta dinámica a partir del año 2018. El artículo 7 de esta misma ley, deja claro, en su letra e), que entre las funciones y atribuciones de las Secretarías Regionales Ministeriales se encuentra la «[promoción de] la vinculación de las actividades de investigación y desarrollo con las necesidades del país, de las empresas y los sectores productivos en las respectivas regiones y fomentar la vinculación público-privada para el desarrollo de iniciativas de ciencia, tecnología e innovación de base científico-tecnológica». Se observa que en Chile claramente se busca implantar un modelo de triple hélice III. Por otra parte, esta ley, mediante su artículo 25, modifica el artículo 9 del Decreto con Fuerza de Ley (DFL) 33, del Ministerio de Educación Pública, de 1981, que crea el Fondo Nacional de Desarrollo Científico y Tecnológico (Fondecyt), el principal organismo de provisión de fondos para la investigación básica en el país. Esta modificación establece que «el financiamiento público de los proyectos y programas establecidos en esta ley tiene como finalidad la creación de nuevo conocimiento científico y tecnológico y la transferencia de tecnología e innovación, aplicando dichos conocimientos en pos del beneficio social y económico, favoreciendo el desarrollo sustentable y el bienestar del país». Podemos apreciar en este objetivo el discurso desarrollista que, como hemos dicho anteriormente, impregna a la transferencia tecnológica. De hecho, en este mis-

7. Por control se entiende la propiedad sobre los resultados de investigación de estas colaboraciones, aunque ciertamente la definición de las líneas de investigación y la rendición de cuentas sobre los gastos es otra forma de entender el «control», una forma que los gobiernos no muy fácilmente ceden. 
mo artículo se da la oportunidad a la institución o a la persona a la que se asignaron los fondos de solicitar la protección mediante instrumentos de propiedad industrial - como la patente- sobre los resultados obtenidos - tanto en proyectos de desarrollo científico como de desarrollo tecnológico- siempre y cuando se otorgue al Estado «una licencia no exclusiva, intransferible, irrevocable y onerosa, respecto de los inventos, innovaciones tecnológicas, diseños o procedimientos» logrados. ${ }^{8}$

Es interesante seguir el proceso de asentamiento de las relaciones de triple hélice apoyado mediante instrumentos jurídicos. Como ejemplo presentamos el relato de Yoda y Kuwashima (2020) en uno de los grandes focos de desarrollo tecnológico, como es Japón. Los autores explican que, en una primera etapa, desde el siglo XIX hasta los años setenta del siglo pasado, la relación industria-universidad era básicamente regional, impulsada a través de los Institutos Regionales de Investigación Pública (IRIP), potenciando las capacidades de producción de la región donde la universidad se encontraba, incluso con apoyo económico y consulta técnica de las empresas. Se entendía que la relación de triple hélice debía desarrollarse en un ambiente libre que fomentase la rápida industrialización de Japón para alcanzar a las potencias occidentales antes de la Segunda Guerra Mundial. No obstante, después de ella y bajo la ocupación estadounidense, el clima jurídico dio un vuelco radical, pues la Ley Antimonopolios, de 1947, impedía la colaboración entre los grandes conglomerados industriales y entre estos, el Gobierno y las universidades. La Ley de Servicio Nacional Público, de 1948, prohibió a los funcionarios públicos, incluyendo los académicos, el trabajo en empresas comerciales mientras ejercieran sus cargos. La Ley de Patentes, de 1959, estableció que las invenciones realizadas por funcionarios públicos pertenecían al Estado. Hasta los años setenta las relaciones entre la industria y la universidad se mantuvieron informalmente, mediante el otorgamiento de becas, las presentaciones en conferencias académicas y el reclutamiento de estudiantes.

La segunda etapa, desde los ochenta a la primera mitad de los noventa, explican Yoda y Kuwashima (2020), se desarrolla a la sombra de los avances en la implantación del modelo de triple hélice impulsado por los Estados Unidos mediante el concepto de parque tecnológico. Silicon Valley fue el modelo a seguir por el gobierno japonés

8. Por otra parte, se establece en ese mismo artículo que «si la institución o persona a la que se le asignaron los recursos logra comercializar en cualquier forma su derecho de propiedad industrial, deberá restituir el $100 \%$ de los fondos asignados, y una suma adicional equivalente al $5 \%$ de los ingresos obtenidos de la comercialización del derecho de propiedad industrial», aunque «el monto que deba restituirse no podrá ser superior al monto recaudado por el asignatario en la comercialización del derecho de propiedad industrial». Dado que originalmente el artículo 9 del DFL 33 consideraba la licencia obligatoria al Estado únicamente para los proyectos de desarrollo tecnológico y que solo había obligación de devolución de los fondos asignados sin una compensación adicional sobre los ingresos, ha habido críticas respecto a esta modificación. Véase Lesly Zurita Arriagada, «La norma que desalienta la creación científica», El Sur, 24 de junio de 2018. 
que aprobó la Ley de Promoción de Zonas Industriales de Alta Tecnología, de 1983. En la práctica, y debido a las restricciones jurídicas imperantes, las disposiciones de esta ley fueron implementadas a través de los IRIP. A pesar de su fortalecimiento, las colaboraciones se consideraban inadecuadas, pues muchas veces no se tomaba en cuenta la verdadera necesidad de los actores industriales. Ese mismo año, el Ministerio de Educación estableció el Sistema de Investigación Conjunta con el Sector Privado, que permitió que las universidades nacionales y los institutos de investigación públicos aceptaran investigadores y fondos de investigación de la industria para realizar investigaciones conjuntas. En este contexto se aprobaron dos leyes que sirvieron de germen a los contemporáneos parques tecnológicos: la Ley de Utilización de Recursos del Sector Privado, de 1986, y la Ley de Ubicación de Instalaciones Clave, de 1988. Bajo estas leyes, se establecieron instituciones de I+D operadas en relaciones industria-Gobierno, que brindaban apoyo industrial. En 1990, la Política de Visión de Tecnología Industrial estableció roles rígidos en las relaciones industria-Gobiernouniversidad: las universidades se dedicarían a la investigación básica, las empresas a la I+D y el Gobierno a la intermediación entre los otros dos actores. De esta manera, y por necesidad de la industria, los IRIP dirigirían sus esfuerzos a la investigación básica y de tecnología de punta.

El tercer período descrito por los autores en referencia, de mediados de los noventa a mediados de la primera década del siglo XXI, es el de desregulación. Su hito principal fue la promulgación de la Ley de Promoción de la Transferencia de Tecnología de las Universidades a los Operadores de Empresas Privadas en 1998, con lo que se establecieron las Oficinas de Licenciamiento Tecnológico (OLT) en las universidades y se permitió el patentamiento universitario. En 1999, se dictó el equivalente nipón de la Ley Bayh-Dole: la Ley de Medidas Especiales para Reactivar la Vitalidad Industrial. Esta ley permitía la apropiación del conocimiento a las instituciones involucradas en contratos de investigación financiados por el Gobierno. La Ley de Mejora de la Tecnología Industrial, del año 200o, permitió a profesores universitarios e investigadores de institutos de investigación nacionales trabajar en emprendimientos que utilizasen tecnologías desarrolladas por ellos. La desregulación continuó, permitiendo a las universidades asociarse en empresas privadas y mixtas, lo que fomentó el desarrollo de la triple hélice. En 1988, las Leyes de Promoción de Zonas Industriales de Alta Tecnología y de Ubicación de Instalaciones Clave fueron reemplazadas por la Ley para Facilitar la Creación de Nuevos Negocios, que permitió brindar no solo apoyo tangible a las empresas mixtas, como el establecimiento de parques tecnológicos, sino también intangible, como otorgar garantías sobre deudas y subvenciones de tecnología a las pequeñas y medianas empresas.

La cuarta etapa, exponen Yoda y Kuwashima (2020) - que cubre el último lustro de la primera década del siglo XXI hasta el presente - es la que siguió a la desregulación. Los IRIP mutan nuevamente, cambiando su objetivo al desarrollo de aplicacio- 
nes prácticas, con lo que parecen volver a su rol inicial. Se derogó la Ley de Utilización de Recursos del Sector Privado, con lo que los parques tecnológicos dejaron de tener apoyo, prefiriendo el modelo de las OLT y de las incubadoras tecnológicas operadas por colaboraciones entre la universidad y el Gobierno. Finalizamos la descripción de este proceso con la aprobación de la Ley de Mejora de la Competitividad Industrial, de 2014, que hizo posible que las universidades actuaran como capitalistas de riesgo.

Aunque para los autores la desregulación sobre la actividad académica ha sido un incentivo gubernamental importante en las relaciones industria-universidad, se entiende que el Gobierno ha actuado únicamente como agente burocrático de control de estas relaciones. Esto puede observarse en el número de patentes solicitadas en relaciones industria-universidad, que es aproximadamente dos órdenes de magnitud más grande que en relaciones de triple hélice.

Una comparación interesante para Chile es la que se puede hacer con un país con una economía en vías de desarrollo. Kruss y Visser (2017) evalúan las relaciones de triple hélice en el contexto de un sistema inmaduro de innovación, como el de Sudáfrica. Al igual que el sistema universitario chileno, el sudafricano puede ser caracterizado como fuertemente diferenciado, segmentado y jerárquico, siendo las principales dominadoras del mercado reputacional aquellas ubicadas en las principales zonas urbanas. Esta estructura impone una barrera no solo a las relaciones con la industria, sino a toda forma de relación; es allí donde es necesaria la intervención gubernamental, ejecutando su rol mediador y facilitador. Los investigadores de universidades prestigiosas tienen menos incentivos a interactuar con la industria y a establecer relaciones de transferencia tecnológica, por lo que debería convencérseles del valor potencial de estas relaciones. Las universidades regionales presentan grandes dificultades en la competencia por la reputación, por lo que estratégicamente deberían alentarse puntos de interacción muy definidos. Este tipo de estrategia también debería funcionar en universidades técnicas, más proclives a la colaboración con la industria, pero muy enfocadas en nichos particulares de investigación. Por tanto, el Gobierno debería establecer una estrategia heterogénea que apoyara a las diferentes universidades en superar las debilidades que presenten.

La teoría dice que la evolución a la triple hélice III es un resultado deseado a nivel mundial en el marco del discurso desarrollista, por lo que se incentiva mediante instrumentos legales. No obstante, ¿qué podemos entender de diferentes experiencias que vayan más allá de la evaluación sobre lo meramente jurídico o a un nivel macro de estudio? En este sentido, y únicamente a manera de ejemplo, podemos referir dos casos europeos de interés. En Portugal, Farinha, Ferreira y Gouveia (2016) reportan el desarrollo de un dispositivo para la recolección de olivas que fue considerado como exitoso en el marco de las relaciones industria-Gobierno-universidad. Una razón de peso es la capacidad de absorción del conocimiento externo por parte de las empresas involucradas en proyectos de triple hélice, lo que implica que estas tengan la 
capacidad de escalar los proyectos para facilitar la comercialización de sus resultados, incluso a nivel internacional. Entre las dificultades encontradas se describe la falta de compromiso completo con el proyecto, tanto por parte de los responsables industriales como de los investigadores universitarios; es más, el Gobierno actúa solo como un agente burocrático, que constriñe rígidamente el accionar del proyecto. Brem y Radziwon (2017) realizan un estudio similar en el proyecto danés GWRP ${ }^{9}$ que derivó de múltiples proyectos de doble hélice a uno de triple hélice. En este caso, la municipalidad de Sønderborg tuvo una participación más activa, lo que permitió escalar el proyecto hasta lograr interés comercial no solo en ese país, sino también en el extranjero. De esta manera, la universidad logró que sus estudiantes pudieran mantenerse involucrados en el proyecto y conseguir más financiamiento para más proyectos de investigación tanto básica como aplicada, la industria tuvo la oportunidad de aprovechar futuras oportunidades comerciales y el Gobierno municipal pudo establecer su región como una de tecnologías ecológicas y así alentar el desarrollo local. Entre los riesgos apreciados se destacan tanto el comportamiento oportunista de actores no involucrados en el ecosistema de desarrollo del proyecto como temas de derechos de propiedad intelectual sobre los resultados de investigación (incluso, la potencial imposibilidad de protegerlos). El acceso a los derechos de propiedad intelectual sobre los resultados de investigación de dichos proyectos es, para la industria, el principal incentivo a participar en esta relación. Sin embargo, la transferencia indirecta al Gobierno y a la universidad de los costos de investigación de la industria, se convierte en un incentivo accesorio de relevancia. Los departamentos de investigación de las universidades que participan en estos proyectos se han convertido en los nuevos departamentos de I+D de la industria (Godin y Gingras, 2000; Barge Gil, Modrego Rico y Santamaría Sánchez, 2006).

\section{Metodología}

Durante el período 2017-2018, se aplicó un cuestionario de 101 preguntas a 918 investigadores universitarios pertenecientes a las siete universidades chilenas que acumulan el 90\% de las patentes provenientes de actividad académica en el periodo 1990-2015. Estos investigadores formaban parte de uno de dos grupos, el de inventores reportados en las patentes otorgadas en el período antes mencionado, o el de investigadores principales de Proyectos Fondef del período 2011-2015. El cuestionario fue respondido por 204 de ellos (24\%), aunque las tasas de respuestas conseguidas en el estado del arte de este tipo de investigaciones no suele ser muy alta - por ejemplo, 27,6\% para Bekkers y Bodas Freitas, 2008; 38\% para Boardman, 2008, Boardman y Corley,

9. Green Water Research Project, un proyecto para utilizar agua de mar profundo como fuente de calor en sistemas de calefacción y refrigeración. 
2008, Boardman y Ponomariov, 2009, y Boardman, 2009; 17,2\% para Grimpe y Fier, 2009; 17\% y 26\% para Haeussler y Colyvas, 2011; 37\% para Klofsten y Jones-Evans, 2000; 24\% para Martinelli, Meyer y Von Tunzelmann, 2008; 38\% para Ponomariov, 2008, y Ponomariov y Boardman, 2008; 14\% para Renault, 2006. Utilizaremos tablas de resumen para presentar los resultados de las ocho preguntas incluidas en el cuestionario que mejor reflejan el estado de la I+D universitaria chilena y su relación con la industria, mediada por la patente como instrumento de transferencia tecnológica.

\section{Resultados}

La tabla 1 muestra la relación en la actividad de transferencia y comercialización del conocimiento entre los investigadores entrevistados y la industria. De ellos, el $70,1 \%$ declara haber realizado trabajos de este tipo con la industria, dedicándole en la actualidad un promedio del $30 \%$ de su tiempo de investigación a proyectos vinculados con la misma. La distribución es sesgada a la derecha, pudiendo apreciarse que el $75 \%$ de los entrevistados dedica 50\% o menos de su tiempo de investigación a proyectos con la industria, mientras el $25 \%$ de ellos lo hace en menos del $20 \%$ de su tiempo de investigación. Por tanto, aunque activa, la relación industria-universidad en la muestra estudiada es minoritaria en cuanto al tiempo dedicado a los esfuerzos de investigación de los académicos.

A pesar de esto, en la tabla 2 se puede observar que cerca del $54 \%$ de la muestra recibe algún tipo de financiamiento industrial, es decir, que aunque la mayoría reporta trabajos de transferencia tecnológica con la industria, esta última ha sido un inversor en la producción del conocimiento transferido en el trabajo de la mitad de los entrevistados.

En relación a lo observado en las dos tablas anteriores, en la tabla 3 se aprecia un apoyo casi absoluto, por parte de los entrevistados, a la potenciación del modelo de triple hélice. Por contra, y a pesar de la actividad y el apoyo a la relación con la indus-

Tabla 1. ¿Ha realizado trabajos con la industria que contribuyan directamente a la transferencia pública-privada o a la comercialización de la tecnología o investigación aplicada?

\begin{tabular}{|lllllllllll}
\hline \multicolumn{10}{c}{ ¿Cuál es el porcentaje de tiempo en investigación } \\
que actualmente Ud. le dedica a proyectos vinculados \\
con la industria?
\end{tabular}


Tabla 2. ¿Su investigación recibe apoyo financiero de la industria?

\begin{tabular}{|lll|} 
& $n$ & $\%$ \\
\hline Sí & 110 & 53,9 \\
No & 94 & 46,1 \\
\hline Total & 204 & 100 \\
\hline
\end{tabular}

Tabla 3. ¿Cree que es importante que se potencie la relación industria-Gobierno-universidad?

\begin{tabular}{lll|} 
& $n$ & $\%$ \\
\hline Sí & 197 & 96,5 \\
No & 5 & 2,5 \\
NC & 2 & 1,0 \\
Total & 204 & 100 \\
\hline
\end{tabular}

tria, los académicos entrevistados se encuentran mayoritariamente en desacuerdo (aproximadamente el 46\%) (tabla 4) con la capacidad de la patente para impulsar el desarrollo. Este resultado podría estar revelando un cierto desconocimiento de los objetivos que persigue la industria al participar, financieramente, de la actividad de $\mathrm{I}+\mathrm{D}$ universitaria, pues los resultados de investigación deben ser trasferibles y para la industria es fundamental que se encuentren protegidos. Se observa un conflicto de intereses entre la academia y la industria, pues, aunque la primera quisiera entrar en relaciones más activas con la segunda, al mismo tiempo duda de la relación causal que se le atribuye a la patente en el desarrollo, tal y como la OMPI lo hace ver con los objetivos ODS.

Nuevamente, podemos observar, a través de la tabla 5, que los investigadores entrevistados dudan de la relación causal entre la propiedad intelectual, en este caso específico la patente, y el desarrollo, pues encuentran mayoritariamente (más del $50 \%$ ) a la patente como un obstáculo al desarrollo de nuevo conocimiento, es decir, exponen que la investigación se complica en áreas en las que el discurso desarrollista de la propiedad intelectual ha permeado.

No obstante, más del 70\% de los entrevistados ha patentado resultados de investigación (figura 8), aunque no necesariamente por alguna obligación causal con la industria, ya que solo $10 \%$ de los que han patentado lo han hecho por este motivo. Encontramos un nuevo punto de aparente incoherencia, pues, aunque no se observa a la patente como una herramienta de impulso del desarrollo, la gran mayoría de los entrevistados ha patentado resultados de su investigación. 
Tabla 4. Con respecto a la afirmación «las patentes son un incentivo necesario para el desarrollo», Ud. se encuentra:

\begin{tabular}{|lll|}
\hline Completamente de acuerdo & $\mathrm{n}$ & $\%$ \\
\hline De acuerdo & 9 & 4,4 \\
\hline Ni de acuerdo ni en desacuerdo & 40 & 19,6 \\
\hline En desacuerdo & 61 & 29,8 \\
\hline Completamente en desacuerdo & 70 & 34,3 \\
\hline Total & 24 & 11,8 \\
\hline
\end{tabular}

Tabla 5. Con respecto a la afirmación «en líneas de investigación proclives al patentamiento se hace muy difícil desarrollar nuevo conocimiento», se encuentra:

\begin{tabular}{|lll|} 
& $\mathrm{n}$ & $\%$ \\
\hline Completamente de acuerdo & 19 & 9,3 \\
De acuerdo & 84 & 41,2 \\
\hline Ni de acuerdo ni en desacuerdo & 63 & 30,9 \\
\hline En desacuerdo & 27 & 13,2 \\
Completamente en desacuerdo & 5 & 2,5 \\
No contesta & 6 & 2,9 \\
Total & 204 & 100 \\
\hline
\end{tabular}

Tabla 6. ¿Ha patentado resultados de investigación?

\begin{tabular}{lllllll} 
& \multicolumn{8}{c}{$\begin{array}{l}\text { ¿Ha patentado por una obligación } \\
\text { contractual de su relación con la } \\
\text { industria? }\end{array}$} \\
& n & $\%$ & Si & $\%$ & No & $\%$ \\
\hline Sí & 129 & 63,2 & 13 & 10,1 & 116 & 89,9 \\
\hline No & 75 & 36,9 & & & & \\
Total & 204 & 100 & & & & \\
\hline
\end{tabular}

Pareciera que, en el caso de la academia chilena, el modelo de producción tecnocientífica es lineal, es decir, se investiga en la universidad, las unidades de transferencia tecnológica buscan que este conocimiento sea patentado por la universidad y, en algunos casos, las patentes son transferidas mediante licenciamiento a la industria, que se encarga de desarrollar la invención patentada y hacerla llegar a la sociedad. 


\section{Conclusiones}

En la raíz del discurso desarrollista de la patente como herramienta de transferencia del conocimiento se encuentran los llamados «efectos secundarios del conocimiento» (knowledge spillover effects), es decir, las externalidades positivas generadas por la creación del conocimiento y que impulsan el emprendimiento mediante start-ups. En su trabajo seminal sobre el tema, Acs y otros (2009) reconocen que, si la protección mediante propiedad intelectual es demasiado fuerte y todas las rentas benefician únicamente al productor de conocimiento, se reducirá la propagación del mismo y, en última instancia, la innovación y el crecimiento. De acuerdo a Hayter (2016), la teoría de Acs y otros se opone a los supuestos teóricos tradicionales de que todo el conocimiento es económicamente útil y que genera efectos secundarios de manera automática. Además, el conocimiento está sujeto a limitaciones institucionales, geográficas y de costos, mientras que la dinámica de su difusión también se ve afectada por sus propiedades: en primer lugar, el valor económico del conocimiento es relativamente incierto; en segundo lugar, el conocimiento se caracteriza por la asimetría en el valor asignado entre los agentes económicos; y en tercer lugar, si bien el costo de transacción para compartir información entre agentes económicos es trivial (Arrow, 1972; Stiglitz, 1999), la transmisión de conocimientos puede requerir una comunicación próxima y a más largo plazo, lo que aumenta los costos de transacción. Por tanto, es de gran importancia plantearse la reflexión de Van Overwalle (2006) sobre cómo reconciliar la tradicional misión académica de producción y diseminación libre del conocimiento con el interés comercial reflejado en el patentamiento del mismo.

En este sentido, Leyden y Link (2015: 80) describen el emprendimiento universitario impulsado por el sector público estadounidense, a través de la Ley Bayh-Dole. Aunque estos autores destacan el impulso que este instrumento jurídico ha dado a la transferencia de conocimiento mediante patentes, señalan que debe entenderse la imposibilidad de incentivar normativamente la innovación; por el contrario, debe asumirse que el conocimiento existe y lo que se quiere es facilitar su patentamiento y licenciamiento. En esta misma línea de pensamiento, Hayter (2016) concluye que las invenciones surgen de años de experiencia en investigación académica y, por lo tanto, son un ejemplo de conocimiento tácito asimilado por profesores y estudiantes, en unidades establecidas a lo largo de un gran proceso temporal. Las patentes son, por tanto, un resultado intermedio que codifica los elementos clave de una invención, pero que no capturan ni pueden capturar por completo el valor total del conocimiento generado durante la investigación académica. Las patentes, incluso las patentes con licencia, tampoco garantizan el intercambio «completo» de conocimientos; esta reflexión apunta a lo expuesto por Wong, Ramos-Toledano y Rojas-Mora (2018), ya que la novedad, como requisito de patentabilidad, incentiva al secretismo en la investigación antes, durante y después de conseguir resultados patentables. 
Los resultados del trabajo de Kruss y Visser (2017) parecen alinearse con los de Hayter (2016), para quien la transmisión de conocimiento técnico depende, principalmente, de la existencia de redes sociales sólidas y capacidades técnicas compartidas entre los diferentes actores. El sistema universitario sudafricano, como ejemplo de un país en vías de desarrollo, no refleja redes de colaboración fuertes entre industria y universidad, tal vez porque las políticas de Gobierno no se enfocan en aquellos nichos que deberían evidenciarlas.

En Chile, otro ejemplo de economía en vías de desarrollo, parece observarse un efecto similar, pues, aunque el Gobierno provee incentivos económicos y fuentes de financiamiento concursables que buscan fomentar las relaciones entre la industria y la universidad, nuestros resultados apuntan a que no logran el objetivo de establecer relaciones formales fuertes. Entre otros actores, la Asociación Chilena de Empresas de Tecnología de Información (2017) expone que los incentivos para los científicos no son adecuados, pues prefieren publicar a patentar, no están inmersos en la «realidad productiva» del país y «no creen que una relación con el empresariado es posible». Además, para este actor el empresariado debería elevar su gasto en I+D desde el $30 \%$ en que se ha mantenido durante el período 2009-2015.

La relación causal entre la propiedad intelectual y el desarrollo, mediado por el desarrollo industrial, que la OMPI señala como la base misma de su misión, encuentra dificultades para ser aceptada en la academia chilena que realiza I+D. Aunque la mayor parte de los investigadores entrevistados ha trabajado en proyectos de transferencia con la industria y ha patentado, no parece que estas actividades estén asociadas con el convencimiento de que la patente, como parte de la propiedad intelectual, sea un motor que contribuya directamente al desarrollo, cuando al mismo tiempo la identifican como un obstáculo para el desarrollo de su quehacer. En conclusión, a pesar de los esfuerzos del Gobierno por establecer un modelo de desarrollo de triple hélice, en Chile el modelo lineal de I+D parece ser el preponderante en las relaciones con la industria, pues la universidad investiga, el Gobierno financia y la industria, con suerte, desarrolla los resultados de investigación patentados.

\section{Referencias}

Acs, Zoltan J., Pontus Braunerhjelm, David B. Audretsch, y Bo Carlsson (2009). «The knowledge spillover theory of entrepreneurship». Small Business Economics, 32 (1): 15-30. DOI: 10.1007/s11187-008-9157-3.

Arrow, Kenneth Joseph (1962). «Economic welfare and the allocation of resources for invention». En Charles K. Rowley (editor), The rate and direction of inventive activity: economic and social factors (pp. 609-626). National Bureau of Economic Research. Princeton: Princeton University Press. 
Asociación Chilena de Empresas de Tecnologías de Información (2017). «Ministerio de Ciencia y Tecnología: ¿qué se espera la nueva institucionalidad?».

BArge Gil, Andrés, Aurelia Modrego Rico y Lluís Santamaría Sánchez (2006). El proceso de transferencia tecnológica universidad-empresa. Barcelona: Fundaciò Empresa i Ciéncia.

BeKkers, Rudi e Isabel Maria Bodas Freitas (2008). «Analysing knowledge transfer channels between universities and industry: To what degree do sectors also matter?» Research Policy, 37 (10): 1.837-1.853. DOI: 10.1016/j.respol.2008.07.007.

BOARDMAN, P. Craig (2008). «Beyond the stars: The impact of affiliation with university biotechnology centers on the industrial involvement of university scientists». Technovation, 28 (5): 291-297. DOI: 10.1016/j.technovation.2007.06.001.

-. (2009). "Government centrality to university-industry interactions: University research centers and the industry involvement of academic researchers». Research Policy, 38 (10): 1.505-1.516. DOI: 10.1016/j.respol.2009.09.008.

BoARDMAN, P. Craig y Elizabeth A. Corley (2008). «University research centers and the composition of research collaborations». Research Policy, 35 (5): 900-913. DOI: 10.1016/j.respol.2008.01.012.

Boardman, P. Craig y Branco Ponomariov (2009). «University researchers working with private companies». Technovation, 29 (2): 142-153. DOI: 10.1016/j. technovation.2008.03.008.

Bradley, Samantha R., Christopher S. Hayter y Albert N. Link (2013). «Models and Methods of University Technology Transfer». Foundations and Trends in Entrepreneurship, 9 (6): 571-650. DOI: 10.1561/0300000048.

Brem, Alexander y Agnieszka Radziwon (2017). «Efficient triple helix collaboration fostering local niche innovation projects-a case from Denmark». Technological Forecasting and Social Change, 123: 130-141. DOI: 10.1016/j.techfore.2017.01.002.

Carayannis, Elias G., Evangelos Grigoroudis, David F. Campbell, Dirk Meissner y Dimitra Stamati (2018). "The ecosystem as helix: an exploratory theory-building study of regional co-opetitive entrepreneurial ecosystems as quadruple/quintuple helix innovation models». R\&D Management, 48 (1): 148-162. DOI: 10.1111/ radm.1230o.

Eтzкоwitz, Henry y Loet Leydesdorff (2000). «The dynamics of innovation: from national systems and "mode 2" to a triple helix of university-industry-government relations». Research Policy, 29 (2): 109-123. DOI: 10.1016/So048-7333(99)00055-4.

FARINHA, Luís, João Ferreira, y Borges Gouveia (2016). «Networks of innovation and competitiveness: A triple helix case study». Journal of the Knowledge Economy, 7 (1): 259-275. DOI: 10.1007/s13132-014-0218-3.

Godin, Benoit (2006). «The linear model of innovation: The historical construction of an analytical framework». Science, Technology \& Human Values, 31 (6): 639-667. DOI: $10.1177 / 0162243906291865$. 
Godin, Benoît y Yves Gingras (200o). «Impact of collaborative research on academic science». Science and Public Policy, 27: 65-73. DOI: 10.3152/147154300781782147.

GrImPE, Christoph y Heide Fier (2009). «Informal university technology transfer: a comparison between the United States and Germany». The Journal of Technology Transfer, 35 (6): 637-650. DOI: 10.1007/s10961-009-9140-4.

Haeussler, Carolin y Jeannette A. Colyvas (2011). «Breaking the ivory tower: Academic entrepreneurship in the life sciences in UK and Germany». Research Policy, 40 (1): 41-54. DOI: 10.1016/j.respol.2010.09.012.

HaYter, Christopher S. (2016). «A social responsibility view of the patent-centric linear model of university technology transfer». Duquesne Law Review, 54: 7-52. Disponible en https://bit.ly/31rdFJH.

Klofsten, Magnus y Dylan Jones-Evans (2000). «Comparing academic entrepreneurship in Europe-the case of Sweden and Ireland». Small Business Economics, 14 (4): 299-309. DOI: 10.1023/A:1008184601282.

KRUss, Glenda y Mariette Visser (2017). «Putting university-industry interaction into perspective: A differentiated view from inside south african universities». The Journal of Technology Transfer, 42 (4): 884-908. DOI: 10.1007/s10961-016-9548-6.

LeYden, Dennis Patrick y Albert N. Link (2015). Public sector entrepreneurship: US technology and innovation policy. Nueva York: Oxford University Press.

Leyden, Dennis Patrick y Matthias Menter (2018). «The legacy and promise of Vannevar Bush: Rethinking the model of innovation and the role of public policy». Economics of Innovation and New Technology, 27 (3): 225-242. DOI: 10.1080/10438599.2017.1329189.

Martinelli, Arianna, Martin Meyer y Nick von Tunzelmann (2008). «Becoming an entrepreneurial university? A case study of knowledge exchange relationships and faculty attitudes in a medium-sized, research-oriented university». The Journal of Technology Transfer, 33 (3): 259-283. DOI: 10.1007/s10961-007-9031-5.

Murray, Fiona y Scott Stern (2007). «When ideas are not free: The impact of patents on scientific research». En Josh Lerner y Scott Stern (editores), Innovation Policy and the Economy, vol. 7 (pp. 648-687). Cambridge, Massachusetts: MIT Press.

ONU, Organización de las Naciones Unidas (2015). «Objetivos de desarrollo sostenible».

OCDE, Organización para la Cooperación y el Desarrollo Económicos (2002). Manual de Frascati: Propuesta para la norma práctica para encuestas de investigación $y$ desarrollo experimental.

Unesco, Organización de las Naciones Unidas para la Educación, la Ciencia y la Cultura (1978). «Recomendación sobre la normalización internacional de las estadísticas relativas a la ciencia y la tecnología».

OMPI, Organización Mundial de la Propiedad Intelectual (2018). «La innovación impulsa el progreso: La OMPI y los Objetivos de Desarrollo Sostenible». 
Ponomariov, Branco y P. Craig Boardman (2008). «The effect of informal industry contacts on the time university scientists allocate to collaborative research with industry». The Journal of Technology Transfer, 33 (3): 301-313. DOI: 10.1007/ s10961-007-9029-Z.

PonOmariov, Branco (2008). «Effects of university characteristics on scientists interactions with the private sector: An exploratory assessment». The Journal of Technology Transfer, 33 (5): 485-503. DOI: 10.1007/s10961-007-9047-x.

Renault, Catherine Searle (2006). "Academic capitalism and university incentives for faculty entrepreneurship». The Journal of Technology Transfer, 31 (2): 227-239. DOI: 10.1007/s10961-005-6108-x.

SACHS, Jeffrey D. y Walter V. Reid (2006). «Environment - Investments toward sustainable development». Science, 312 (5.776): 1.002. DOI: 10.1126/science.1124822.

SII, Servicio de Impuestos Internos (2016). «Oficio 1706. Régimen Tributario de Científicos, Innovadores e Investigadores Internacionales».

Siegel, Donald S., David A. Waldman, Leanne E. Atwater y Albert N. Link (2004). «Toward a model of the effective transfer of scientific knowledge from academicians to practitioners: Qualitative evidence from the commercialization of university technologies». Journal of Engineering and Technology Management, 21 (1-2): 115-142. DOI: 10.1016/j.jengtecman.2003.12.006.

Stiglitz, Joseph E. (1999). «Knowledge as a global public good». En Inge Kaul, Isabelle Grunberg y Marc Stein (editors), Global Public Goods: International Cooperation in the 21st Century, (pp. 308-25). New York: Oxford University Press.

Thursby, Jerry G., Richard Jensen y Marie C. Thursby (2001). «Objectives, characteristics and outcomes of university licensing: A survey of major US universities». The Journal of Technology Transfer, 26 (1-2): 59-72. DOI: 10.1023/A:1007884111883. VAN Overwalle, Geertrui (2006). «Reconciling patent policies with the university mission». Ethical Perspectives, 13 (2): 231-247. DOI: 10.2143/EP.13.2.2016632.

Wong, Sulan, Joan Ramos-Toledano y Julio Rojas-Mora (2018) «Sobre la in-compatibilidad de la ciencia abierta con la novedad como estándar de patentabilidad». Jurídicas, 15 (2): 88-103. DOI: 10.17151/jurid.2018.15.2.6.

YoDA, Noriko y Kenichi Kuwashima (2020). «Triple helix of university-industry-government relations in Japan: Transitions of collaborations and interactions». Journal of the Knowledge Economy, 11 (3): 1.120-1.144. DOI: 10.1007/s13132-019-00595-3.

Yu, Peter K. (2016). «Five decades of intellectual property and global development». The WIPO Journal, 8 (1): 1-10. Disponible en https://bit.ly/3pouBJb.

Ziman, John (2002). Real science: What it is and what it means. Cambridge: Cambridge University Press. 


\section{Agradecimientos}

El presente trabajo ha sido financiado parcialmente por el proyecto ANID/FONDECYT 11150162 y por el proyecto DIUFRO DI19-0043.

\section{Sobre los autores}

Sulan Wong es profesora asociada del Departamento de Ciencias Jurídicas de la Universidad de La Frontera, Chile. Obtuvo su título de abogada en la Universidad de Los Andes, Venezuela. Doctora en Derecho por la Universidad de La Coruña, España. Ha tenido estancias de investigación en la Universidad de Barcelona, España, en la Universidad de Aviñón, Francia, y el Institut National de Recherche en Informatique et en Automatique, Francia. Miembro del Centro de Investigación sobre Desafíos Internacionales de la Universidad de La Frontera. Su principal línea de investigación se dedica al estudio de la interacción entre la ciencia, la tecnología y la propiedad intelectual. Su correo electrónico es sulan.wong@ufrontera.cl. (D) https:// orcid.org/oooo-0003-1684-0285.

Julio Rojas-Mora es profesor asistente del Departamento de Ingeniería Informática de la Universidad Católica de Temuco, Chile. Obtuvo su título de Ingeniero de Sistemas en la Universidad de Los Andes, Venezuela, y un doctorado del Departamento de Economía y Organización de Empresas de la Universitat de Barcelona, España. Ha realizado estancias de investigación tanto en la Université d'Avignon como en el Institut National de Recherche en Informatique et en Automatique de Francia. Además, hizo un postdoctorado en el Laboratorio ESPACE del Centre National de la Recherche Scientifique del mismo país. Su principal línea de investigación se basa en el estudio de métodos para el apoyo de toma de decisiones en entornos inciertos. Su correo electrónico es jrojas@inf.uct.cl. (D) https://orcid.org/oooo-00o2-0268-8748. 


\title{
REVISTA CHILENA DE DERECHO Y TECNOLOGÍA
}

La Revista Chilena de Derecho y Tecnología es una publicación académica semestral del Centro de Estudios en Derecho Informático de la Facultad de Derecho de la Universidad de Chile, que tiene por objeto difundir en la comunidad jurídica los elementos necesarios para analizar y comprender los alcances y efectos que el desarrollo tecnológico y cultural han producido en la sociedad, especialmente su impacto en la ciencia jurídica.

\author{
EDITOR GENERAL \\ Daniel Álvarez Valenzuela \\ (dalvarez@derecho.uchile.cl) \\ SITIO WEB \\ rchdt.uchile.cl \\ CORREO ELECTRÓNICO \\ rchdt@derecho.uchile.cl \\ LICENCIA DE ESTE ARTÍ́CULO \\ Creative Commons Atribución Compartir Igual 4.o Internacional
}

\begin{abstract}
s
La edición de textos, el diseño editorial

y la conversión a formatos electrónicos de este artículo

estuvieron a cargo de Tipográfica

(www.tipografica.io).
\end{abstract}

